\title{
Meritocracia y desigualdad económica: Percepciones, preferencias e implicancias.
}

Meritocracy and economic inequality: Perceptions, preferences and implications.

(Pre-print, aceptado en Abril 2018 en Revista Internacional de Sociología)

Juan C. Castillo ${ }^{1}$, Alex Torres ${ }^{2}$, Jorge Atria ${ }^{1} \&$ Luis Maldonado $^{1}$

${ }^{1}$ Institute of Sociology, Pontificia Universidad Católica, Chile

${ }^{2}$ Assessment Center Mide UC, Pontificia Universidad Católica, Chile

Contact: jcastillov@uc.cl, jc-castillo.com

\section{Funding}

This work was supported by the National Fund for Economic and Scientific Development FONDECYT, grant number 1160921, as well as by the Centre for Conflict and Cohesion Studies COES, CONICIT/FONDAP/15130009. 
Meritocracia y desigualdad económica: Percepciones, preferencias e implicancias. Meritocracy and economic inequality: Perceptions, preferences and implications.

\title{
Resumen
}

La idea de meritocracia está relacionada con la distribución de bienes y beneficios basados en el talento y esfuerzo individual, constituyendo un principio que legitima la distribución desigual de los recursos en sociedades modernas. Pese a las constantes referencias a la meritocracia en la investigación de desigualdad y estratificación social en sociología, existen pocos intentos conceptuales y empíricos que busquen entender hasta qué punto los individuos perciben y prefieren la meritocracia, así como también sus consecuencias. La presente investigación propone un marco conceptual y empírico para el estudio de las percepciones y preferencias meritocráticas, que posteriormente es relacionado con una serie de variables relacionadas con desigualdad social. Utilizando datos de la encuesta chilena "Justicia social y participación ciudadana" ( $\mathrm{N}=1.245)$, los resultados del análisis sugieren que las preferencias y percepciones de meritocracia son constructos distintos y que quienes perciben un adecuado funcionamiento de la meritocracia también perciben menos desigualdad social.

Palabras Clave: Meritocracia, desigualdad económica, percepción de desigualdad, estatus social, justicia distributiva.

\begin{abstract}
The idea of meritocracy is related to the distribution of goods and rewards based on individual talent and effort, being a principle that legitimizes the unequal distribution of resources in modern societies. Despite the constant references to meritocracy in inequality and stratification research, there are still few conceptual and empirical attempts to understand to what extent individuals perceive and prefer meritocracy, as well as their consequences. The present research proposes a conceptual and empirical framework for studying meritocratic perceptions and preferences, which is then related with economic inequality variables. Using data from the Chilean survey "Social justice and citizenship participation", the analyses suggest that meritocratic perceptions and preferences are different constructs and that those perceiving an adequate functioning of meritocracy also perceive less economic inequality.
\end{abstract}

Keywords: Meritocracy, economic inequality, inequality perception, social status, distributive justice. 


\section{Introducción}

El interés en el fenómeno de la desigualdad económica se ha incrementado notoriamente en los últimos años. Las crisis financieras, los movimientos sociales y las demandas por mayor redistribución de recursos alrededor del mundo han motivado a los científicos sociales de distintas disciplinas a centrarse en el estudio tanto de aspectos objetivos como subjetivos de la desigualdad. Tras esta agenda se encuentra una paradoja: ¿Cómo es posible que los individuos en sociedades democráticas puedan convivir con extremos niveles de desigualdad? El supuesto a la base de esta pregunta es que la igualdad es un valor central y consensual en las democracias modernas, y que por lo tanto la desigualdad sería una amenaza para ella (Lipset, 1959). Sin embargo, aún cuando sería esperable que en sociedades con mayor desigualdad existieran mayores presiones hacia la redistribución económica (Meltzer y Richard, 1981), la evidencia sugiere que esto no necesariamente ocurre en la práctica (Schröder, 2017; Solt, 2010; Solt et al., 2016). En este sentido, el estudio de distintas formas de justificación de la desigualdad económica cobra especial importancia a la hora de entender la relación entre desigualdad e injusticia (Castillo, 2012) y las acciones que se toman en torno a ellas.

Desde las ciencias sociales se ha señalado que el advenimiento de la modernidad ha tenido como una de sus implicancias la necesidad de generar mecanismos concretos y simbólicos que permitan y expliquen la asignación de los recursos de forma desigual, pero sin recurrir a elementos característicos del ancien régime como herencias o títulos honoríficos (Tocqueville, 2004). Los ideales de igualdad que se afirman principalmente desde la Ilustración comienzan entonces a socavar la legitimidad de características adscriptivas como bases para la distribución, abriendo espacio a criterios de mérito individual como el esfuerzo y el talento (Hadjar, 2008; Young, 1962). Así, la meritocracia se puede definir de manera general como la idea -implícita o explícita- de que se recibirá una compensación proporcional a nuestros méritos, mientras la conducta se mantenga dentro de los límites de la estructura jurídica y de lo socialmente aceptado (Bell, 1977; Young, 1962). Este principio de asignación serviría para legitimar la jerarquía de estatus presente en las democracias industriales modernas, fomentando la idea de ser sociedades que respetan y promueven la justicia social, en contraste con las dominancias coercitivas o heredadas de las élites en las sociedades tradicionales previas (Warikoo y Fuhr, 2014).

En la sociología el concepto de meritocracia posee un lugar central en estudios de estratificación y movilidad social. Sociedades más móviles se caracterizarían por un menor grado de determinación familiar del estatus individual, dejando por tanto mayor espacio al mérito individual como factor determinante de la posición social alcanzada. Sin embargo, la 
investigación sociológica a la fecha en este tema ha estado caracterizada principalmente por estudios de movilidad social y sus críticas a la implementación del ideal meritocrático (Goldthorpe, 2003), abordando escasamente lo que las personas perciben y creen respecto de la meritocracia. La presente investigación tiene como primer objetivo detenerse precisamente en este punto, centrándose en el concepto de meritocracia y en su evaluación en base a lo que las personas manifiestan percibir y creer. La pregunta asociada a este objetivo se puede plantear como: ¿Es posible distinguir percepciones y creencias en meritocracia? Hasta ahora, la aproximación a la medición de meritocracia en sociología ha sido mediante la utilización ad-hoc de indicadores disponibles en datos secundarios, sin abordar aspectos de la validez de estas mediciones de manera sistemática (Duru-Bellat y Tenret, 2012; Reynolds y Xian, 2014). En este marco, una primera hipótesis a contrastar en esta investigación es que es necesario y posible distinguir entre percepción (lo que es) y preferencias (lo que debería ser) sobre meritocracia, y que ambos elementos deben ser tomados en consideración en conjunto para poder avanzar en una agenda de investigación sobre este tema.

Además de proponer una distinción conceptual y de medición de percepciones y preferencias respecto de meritocracia, un segundo objetivo a abordar corresponde a las diferencias individuales en términos de estatus socioeconómico. ¿Existen diferencias de estatus en percepciones y preferencias de meritocracia? Siguiendo una tesis racional es plausible plantear como hipótesis que aquellos de mayor estatus (asociado a ingreso y educación) percibirán y preferirán más la meritocracia como una forma de legitimar su posición en la sociedad, mientras que sucedería lo opuesto para aquellos de menos estatus. Junto a ello, un tercer objetivo en esta investigación es indagar sobre las posibles implicancias de la meritocracia, en particular en la percepción de desigualdad económica: ¿Cómo se relacionan percepciones y preferencias de meritocracia con la percepción de desigualdad económica? Dado que la meritocracia sería una forma de legitimación de desigualdad en relación a criterios de esfuerzo y talento, se propone como hipótesis que aquellos que perciben que la meritocracia funciona adecuadamente tenderán a minimizar su percepción de desigualdad. Es decir, la desigualdad se vuelve menos notoria (y probablemente menos relevante) para aquellos que consideran que el sistema distributivo basado en principios meritocráticos opera correctamente.

Los objetivos e hipótesis de investigación se resumen en el diagrama de la Figura 1. En el centro aparece el concepto de meritocracia y la distinción entre sus dos principales componentes, cuya operacionalización y medición como variables latentes corresponde al primer objetivo. Luego, a la izquierda aparecen los factores de estatus social asociados a meritocracia que constituyen el segundo objetivo, mientras la relación entre meritocracia y percepción de desigualdad se enmarca en el tercer objetivo de esta investigación. Es mediante 
las asociaciones con status y percepción de desigualdad que se pretende avanzar en los vinculos entre meritocracia y desigualdad económica.

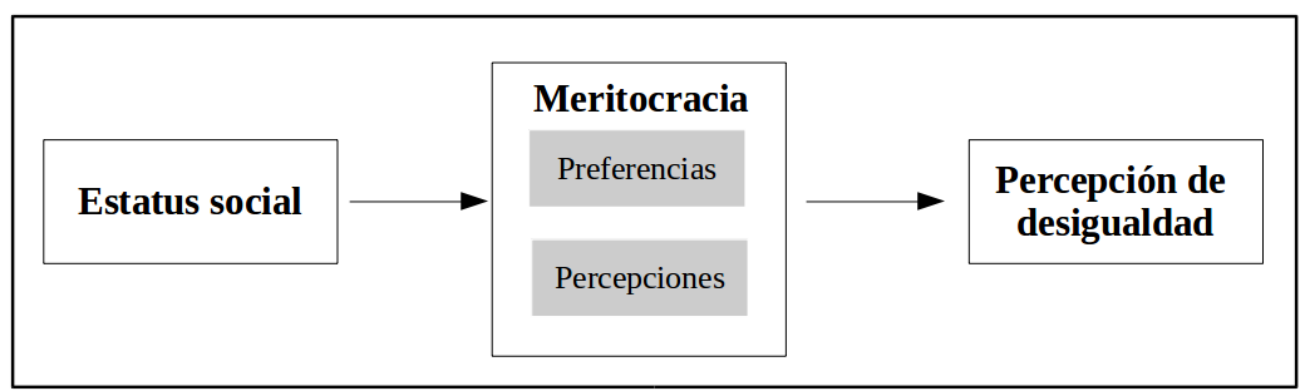

Figura 1: Conceptos centrales y su asociación

En lo que sigue, la primera sección comienza abordando los aspectos conceptuales y la evidencia empírica relacionada con el estudio de la meritocracia. La segunda parte se refiere a una serie de factores asociados a la meritocracia, como el estatus socioeconómico y la percepción de desigualdad económica. Luego de presentar los datos, variables y métodos, se resumen los principales resultados del análisis y finalmente se discuten implicancias para futuras investigaciones.

\section{Perspectivas en el estudio de la meritocracia}

Hasta ahora hemos hablado de meritocracia sin hacer referencia a una clara definición conceptual del término. Esto no es casual ya que existe gran ambigüedad en la literatura al respecto, lo que es reconocido por Sen al señalar que "la idea de la meritocracia puede tener muchas virtudes, pero la claridad no es una de ellas" (Sen, 2000: 5). Preliminarmente, constatamos que una acepción general se refiere a la caracterización de un orden social donde "las recompensas son distribuidas a los individuos de acuerdo con criterios de mérito personal" (Yair, 2007: 1), denotando que lo central de la meritocracia es un orden donde el mérito prima como criterio de distribución, y por lo tanto "la meritocracia se contrasta también con sistemas que estan basados en la selección mediante características adscriptivas como riqueza heredada, clase social, etnicidad, raza y, más generalmente, con cualquier sistema de nepotismo" (Yair, 2007: 1). Esto nos conduce a enfocarnos en la definición de mérito para poder entender qué es meritocracia, aún cuando tal como sucede con la meritocracia, la definición de mérito es generalmente ambigua, y en la mayoría de lo casos simplemente ausente. Una de las pocas definiciones centrales e influyentes al respecto es la aportada por Young (1962), quien acuña el término meritocracia en su novela satírica "The 
rise of meritocracy". En ella, mérito se define formalmente como $M$ (mérito) $=I$ (inteligencia o talento $)+E($ esfuerzo $)$. La consideración de esfuerzo y talento como componentes del mérito cuenta con cierto consenso en la literatura, aún cuando "una deficiencia seria en los fundamentos éticos de la meritocracia es su virtual ausencia de discurso respecto a qué áreas del "mérito" son las más (o menos) recompensadas" (Horowitz, 2006: 131). De esta manera, algunos autores enfatizan los aspectos relacionados con esfuerzo por sobre el talento, o viceversa.

Teniendo en cuenta esta definición básica de mérito y meritocracia, es posible proponer un marco general de estudio donde luego situar la presente investigación. Este marco se compone de tres perspectivas principales, que denominaremos normativa, descriptiva y subjetiva. La perspectiva normativa se asocia disciplinarmente a la filosofía política, caracterizada por la discusión respecto de la justicia y sus principios (Krebs, 2000; Rawls, 1971; Sandel, 1998). En este marco se plantea la pregunta más amplia respecto de lo justo y de los criterios distributivos que pueden ser catalogados como tal. En este marco la meritocracia aparece vinculada a la justicia como equidad (Aristotle, 1999; Homans, 1974) o igualdad proporcional en este caso a talento y esfuerzo, así como también a otros criterios de merecimiento que podrían competir con la meritocracia. Aún cuando se podría decir que en las sociedades modernas el mérito es considerado en general un criterio justo de distribución, también es posible identificar otras situaciones o esferas (Walzer, 1983) donde normas basadas en principios de igualdad o necesidad pueden primar como criterio de justicia (Deutsch, 1975). Esta discusión sobre los principios distributivos justos y sobre el tema del merecimiento (deservnigness) va más allá de los objetivos de este artículo, pero sin duda es relevante de mencionar dadas las posibles implicancias de la investigación sociológica en la tradición normativa, así como también porque sirve como insumo a la perspectiva subjetiva, como es descrito más adelante.

Así como la perspectiva normativa es más cercana disciplinarmente a la filosofía política, la perspectiva descriptiva es de corte más sociológico y se interesa principalmente en la implementación del ideal meritocrático vía estudios de movilidad social (Alon y Tienda, 2007; Duru-Bellat y Tenret, 2012; Goldthorpe, 2003). En este sentido se asume que, idealmente en sociedades meritocráticas modernas, las posiciones estarían abiertas y vinculadas principalmente a credenciales educacionales que reflejan criterios de mérito y no al estatus social de origen familiar. De esta manera, en un contexto de igualdad de oportunidades, la meritocracia generaría un alto grado de movilidad social. La mayor parte de la literatura en esta perspectiva descriptiva se caracteriza por una aproximación crítica a la implementación del ideal meritocrático: la meritocracia no conllevaría movilidad sino que una mera reproducción del estatus ya existente. En este proceso, la educación ocuparía una 
función clave, pues a través de ella se legitimaría la meritocracia (a quiénes más estudian mayores recompensas les esperan) mientras simultáneamente se reproduce el estatus en una sociedad (Bourdieu y Passeron, 1990; Warikoo y Fuhr, 2014). Este efecto reproductor se produce en la medida que aquellos más "inteligentes y trabajadores" logran subir en la jerarquía social, lo que generaría desigualdades en el acceso a las oportunidades por parte de sus descendientes (Page, 2013; Young, 1962).

La evidencia empírica sobre el debate meritocracia-igualdad de oportunidades es en su mayoría consistente con las críticas de carácter reproduccionista, lo que viene a poner en entredicho la capacidad de adjudicar justamente los recursos de las sociedades en base a criterios meritocráticos (Breen y Goldthorpe, 2001; Goldthorpe, 2003). Por ejemplo, las investigaciones de Piketty señalan que los aumentos de salarios en deciles superiores no necesariamente se relacionan con mejores calificaciones, idea que también se refuerza en el Informe sobre Desigualdad en el Mundo (Oxfam, 2016).

La perspectiva normativa y descriptiva en el estudio de la meritocracia sirven como referente y contexto para introducir ahora la perspectiva subjetiva, tal como se representa esquemáticamente en la Figura 1:

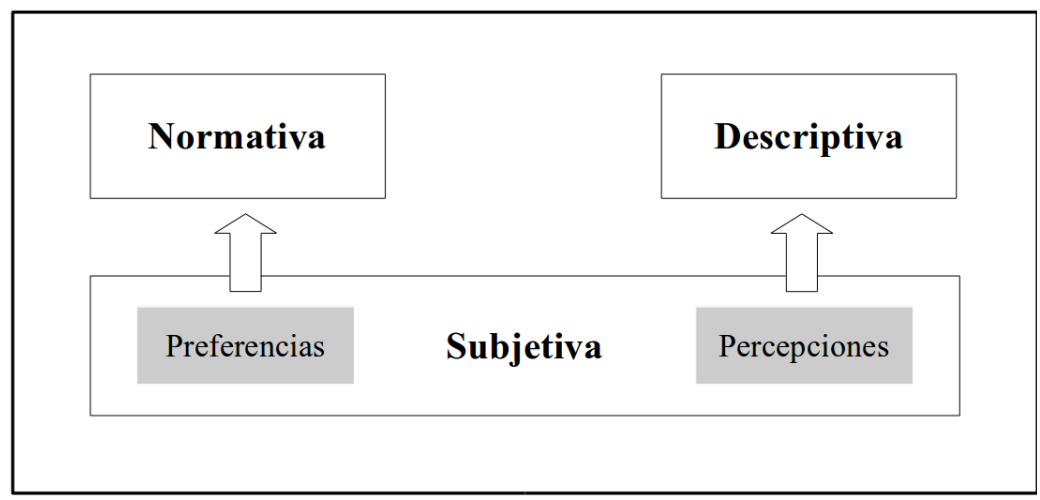

Figura 2: Perspectivas en el estudio de la meritocracia

En la Figura 1 es posible identificar arriba las dos perspectivas identificadas anteriormente en relación al estudio de la meritocracia: normativa (filosofía política) y descriptiva (sociología de la desigualdad/estratificación). A estas se suma la perspectiva subjetiva, que contiene dos elementos principales y además vinculados con las dos perspectivas anteriores: las preferencias, en relación a los principios normativos, y la percepción del funcionamiento de la meritocracia. Así, en esta perspectiva subjetiva se reflejan de cierta manera las perspectivas normativas (preferencias respecto de lo que debería 
ser) y descriptivas (percepciones de lo que es), conceptos que se detallan en la sección siguiente.

\section{La perspectiva subjetiva en el estudio de la meritocracia}

El comienzo de las investigaciones de los aspectos subjetivos de la desigualdad económica en sociología puede ser rastreado a fines de los años 1940s, vinculado a conceptos como deprivación relativa y teoría de la equidad (Adams, 1963; Stouffer et al., 1949). Sin embargo, no es hasta la publicación de Kluegel y Smith sobre creencias sobre la estratificación (1981) que se logra identificar claramente un ámbito de estudios relacionado con aspectos subjetivos sobre desigualdad y sus factores asociados al interior de la sociología. El listado de publicaciones a la fecha en esta línea es numeroso y abarca una gran diversidad de temas y metodologías ${ }^{1}$, dentro de los cuales el estudio de la meritocracia representa sin embargo un ámbito bastante limitado. Las pocas investigaciones en relación a aspectos subjetivos de la meritocracia tienden a focalizarse principalmente en las preferencias de la meritocracia, que se refiere a si la meritocracia es internalizada como un principio deseado para generar justicia, es decir, si los sujetos adhieren a la idea de que el mérito debe ser recompensado (Foster y Tsarfati, 2005; Jaime-Castillo y Marqués-Perales, 2014; Son Hing et al., 2011). Por ejemplo, esta preferencia se puede expresar en el grado de acuerdo con afirmaciones como "las personas que se esfuerzan más deberían obtener mayores salarios".

Por su parte, la percepción de meritocracia se refiere a cómo los sujetos captan las diferencias sociales presentes en su entorno y en qué medida atribuyen que éstas son el resultado de un sistema meritocrático y de sus elementos constitutivos de trabajo y habilidad (Duru-Bellat y Tenret, 2012; Foster y Tsarfati, 2005: Reynolds y Xian, 2014). . Contrastando con la preferencia, la percepción se relaciona por ejemplo con frases como "las personas que se esfuerzan más obtienen mayores salarios". De manera resumida, la percepción tiene que ver con lo que es observado (lo que es), mientras la preferencia con lo que a las personas les gustaría que ocurriera (lo que debería ser). Respecto de este contraste entre percepción y preferencia, Duru-Bellat y Tenret (2012) han desarrollado el único estudio bajo nuestro conocimiento que distingue percepciones y preferencias en la meritocracia, con datos del International Social Survey Programme (ISSP) 1999 comparando 26 países. Sin embargo, ellos solo utilizan un indicador para cada concepto, sin detenerse en la discusión sobre el error de medición que esta estrategia conlleva, tema que detallaremos más adelante.

1 Para una revisión consultar Aalberg (2003) y Janmaat (2013) 
La distinción conceptual entre percepción y preferencia en la meritocracia nos parece fundamental para poder avanzar en la comprensión e investigación de este fenómeno por varias razones. En primer lugar esta la distinción de ambas dimensiones permitiría ahondar en el estudio de las posibles asociaciones y diferencias que existirían entre el nivel de meritocracia que los individuos perciben en una sociedad y el nivel en que desearían que ella funcionara como sistema de distribución. Y en segundo lugar, la confusión entre estos conceptos podría llevar a conclusiones erróneas, como por ejemplo que un bajo nivel de meritocracia percibido necesariamente se asocie a una menor preferencia en la meritocracia como ideal, o viceversa. Por lo tanto, en la presente investigación una primera hipótesis (H1) señala que la meritocracia en su dimensión subjetiva presenta una estructura que permite diferenciar aspectos descriptivos (percepción) y normativos (preferencias). En el marco de una metodología cuantitativa, un supuesto de esta hipótesis, es que los aspectos de percepción y de preferencias en la meritocracia son posibles de medir basados en indicadores de encuestas, que a su vez sirvan de insumo a análisis confirmatorios de la estructura conceptual propuesta. El contraste satisfactorio de esta hipótesis de medición permitiría contar con instrumentos de medición que puedan luego servir como insumos para investigaciones posteriores.

\section{Factores asociados a las percepciones y preferencias en la meritocracia}

Un primer elemento a relevar en relación al estudio de percepciones y preferencias en la meritocracia es el del estatus social. Investigaciones previas han observado que la posición que las personas ocupen en la estructura social afecta la forma en que se explican las desigualdades y en particular la importancia que se le da al mérito (Duru-Bellat y Tenret, 2012; Solt et al., 2016). En este sentido, las personas que pertenecen a los grupos dominantes de una sociedad o que se encuentran en la parte superior de la estructura social explican tal posición comúnmente por cualidades personales o factores que están bajo su propio control, como sus habilidades o el trabajo invertido (Lerner, 1980), mientras que las personas en situación de dominación o desventaja refieren a factores fuera de su control para explicarla. Sin embargo, algunas teorías más cercanas a la psicología social han planteado una perspectiva alternativa, donde aquellos en situación de bajo estatus son los que más sostienen preferencias que tienden a mantener o justificar su situación de desventaja en un sistema social. Esto ocurriría dada la necesidad de justificar y defender el status quo de los sistemas sociales mediante la creencia de que ciertos grupos sociales merecen su lugar en la jerarquía social (Jost et al., 2003), en este caso particular debido a sus habilidades y esfuerzo, aunque esta creencia o percepción no los favorezca individualmente. No obstante, y acorde con la evidencia de investigaciones ligadas más cercanamente a la sociología y al rol de variables de estatus en creencias respecto a la distribución (Kunovich y Slomczynski, 2007) nuestra 
hipótesis inicial en este ámbito (H2) es que aquellos que poseen mayor estatus social, presentarán mayores niveles de percepción de meritocracia, así como mayores niveles de adhesión a la preferencia meritocrática.

Dentro de las variables de estatus la educación requiere una consideración especial, dado que se han argumentado y evidenciado efectos a veces opuestos en actitudes hacia temas redistributivos (Schneider y Castillo, 2015). Por un lado, se señala que aquellos con altos niveles de educación formal podrían presentar mayores niveles de adscripción a las preferencias meritocráticas (Baer y Lambert, 1982; Bourdieu y Passeron, 1990), dado que la meritocracia sería una ideología dominante y la educación formal sería una forma de socialización en este tipo de ideologías. Sin embargo, Baer y Lambert (1982) señalan que la educación puede comportarse de forma opuesta, dado que las personas más educadas de una sociedad serían más conscientes de las inequidades de la realidad en que viven y de los factores que influyen en ella, y de este modo, creerían y/o percibirían menos meritocracia en su entorno. Para la presente investigación se asumirá como hipótesis la concordante con la primera de las dos corrientes, es decir, mayores niveles educacionales presentarán mayores niveles de percepción de la meritocracia, así como mayores niveles de apoyo a las preferencias meritocráticas.

Además de las variables de estatus, un segundo aspecto a indagar en este estudio es el de la relación entre meritocracia y percepciones de desigualdad económica. Una de las características de las preferencias en la meritocracia es que permite a las personas explicarse las jerarquías de estatus, es decir, ejecuta una función de legitimación o justificación de las diferencias y desigualdades dentro de una sociedad (Hadjar, 2008). Al respecto, una primera posibilidad es que la preferencia por la meritocracia funcione como una ideología consensualmente compartida por los miembros del sistema social que justifica la distribución de bienes o elementos valorados positivamente (Lane, 1986). En especifico, consistiría en un mito legitimador promotor de la jerarquía social, ya que proveería justificaciones morales y racionales para la opresión y la desigualdad social, las que serían sostenidas por las personas debido a una tendencia general a ordenar a la sociedad en grupos jerárquicos (Costa-Lopes et al., 2013). Una segunda forma de entender la relación entre meritocracia y justificación de la desigualdad es la del interés racional, que plantea que quienes se encuentran en una situación actual de privilegio presentarían mayores niveles de preferencia y percepción de meritocracia como una forma de justificar su actual estatus en base a su esfuerzo y su intelecto (Kunovich y Slomczynski, 2007). Esto podría generar consecuencias en términos de percepción de desigualdad económica en una sociedad, ya que ésta se encontraría justificada como fruto del 
mérito. En esta línea, se plantea como hipótesis (H3) que aquellos que perciban y prefieran más la meritocracia percibirán a su vez una menor desigualdad económica.

\section{Datos, variables y métodos}

\section{Datos}

La presente investigación utilizó los datos generados por el proyecto "Desigualdad, Justicia Social y Participación Ciudadana" (Proyecto FONDECYT ${ }^{2}$ 11121203), el que mediante encuestas personales aplicadas en hogares el año 2013 recogió información sobre temas referidos a desigualdad y justicia social. En el diseño muestral se consideró como universo a la población mayor de 18 años de las comunas de Chile que cuentan con más de 100.000 habitantes según el censo realizado el año 2002. Se realizó un muestreo probabilístico estratificado en 4 etapas, con afijación para la muestra en la Región Metropolitana. Respecto de las etapas del muestreo estratificado, en primer lugar se seleccionaron comunas aleatoriamente mediante el procedimiento Probabilities Proportionate to Size, otorgando a 46 comunas una probabilidad de 1 de ser elegido, quedando representadas 13 de las 15 regiones del país. En segundo lugar, dentro de las comunas ya seleccionadas, se seleccionaron manzanas mediante muestreo aleatorio simple, para luego determinar una vivienda y un habitante de la vivienda mayor a 18 años en la tercera y cuarta etapa. Se sobredimensionó la muestra estimando una tasa de respuesta de 80\%, obteniéndose finalmente 1245 casos, lo que está sobre el número establecido de la muestra de 1200 participantes. En todos los casos se contó con el consentimiento informado de los participantes.

\section{Variables}

El primer objetivo de este trabajo dice relación con la medición de dos aspectos de la meritocracia: percepción y preferencias. Para cada uno de ellos se consideran tres indicadores del cuestionario, los de percepción con un énfasis descriptivo de cómo los participantes ven que la meritocracia opera en la sociedad, mientras los de preferencias poseen un énfasis normativo con criterios de evaluación sobre lo que estaría bien y/o debería ser. En los análisis a presentar posteriormente se busca establecer la presencia de los dos factores subyacentes a este set de indicadores, los que luego serán utilizados como variables (latentes) en los modelos de regresión. Los indicadores se resumen en la Tabla 1.

2 Fondo Nacional de Desarrollo Científico y Tecnológico (Chile) 
Tabla 1: Indicadores de percepciones y preferencias sobre meritocracia

\begin{tabular}{|c|c|c|c|}
\hline Dimensión & Ítems & Respuesta & Descriptivos \\
\hline \multirow{4}{*}{$\begin{array}{l}\text { Percepción de } \\
\text { Meritocracia }\end{array}$} & $\begin{array}{l}\text { 1. En Chile las personas son recompen- } \\
\text { sadas por sus esfuerzos. }\end{array}$ & \multirow{7}{*}{$\begin{array}{l}\text { 1. Muy en desacuerdo } \\
\text { 2. En desacuerdo } \\
\text { 3. Ni de acuerdo ni } \\
\quad \text { en desacuerdo } \\
\text { 4. De acuerdo } \\
\text { 5. Muy de acuerdo }\end{array}$} & $\begin{array}{l}\mathrm{M}=2.41 \\
\mathrm{SD}=1.01\end{array}$ \\
\hline & 2. En Chile las personas obtienen lo que & & $\mathrm{M}=2.30$ \\
\hline & merecen. & & $\mathrm{SD}=0.96$ \\
\hline & $\begin{array}{l}\text { 3. En Chile las personas son recompen- } \\
\text { sadas por su inteligencia y habilidades. }\end{array}$ & & $\begin{array}{l}\mathrm{M}=2.70 \\
\mathrm{SD}=1.05\end{array}$ \\
\hline \multirow{3}{*}{$\begin{array}{l}\text { Preferencias por } \\
\text { la meritocracia }\end{array}$} & $\begin{array}{l}\text { 1. Está bien que haya personas que tie- } \\
\text { nen más dinero o riqueza, pero sólo si } \\
\text { hay igualdad de oportunidades. }\end{array}$ & & $\begin{array}{l}\mathrm{M}=3.91 \\
\mathrm{SD}=0.93\end{array}$ \\
\hline & $\begin{array}{l}\text { 2. Las personas tienen derecho a mante- } \\
\text { ner o conservar lo que han ganado, aun } \\
\text { cuando eso signifique que algunas per- } \\
\text { sonas sean más ricas que otras. }\end{array}$ & & $\mathrm{M}=3.77$ \\
\hline & $\begin{array}{l}\text { 3. Las personas que trabajan duro me- } \\
\text { recen ganar más que las que no lo ha- } \\
\text { cen }\end{array}$ & & $\mathrm{M}=4.11$ \\
\hline
\end{tabular}

El segundo set de variables corresponde a estatus socioeconómico y se presentan en la Tabla 2. Para ello se consideran variables de ingreso y educación, y además se incluye una medida de estatus subjetivo como complemento para el análisis. En el caso de la variable de ingreso, para obtener el ingreso familiar per cápita se dividió el ingreso del hogar por el número de integrantes reportados. En los modelos se utiliza un término logarítmico para esta variable. 
Tabla 2: Variables Independientes de estatus socioeconómico

\begin{tabular}{|c|c|c|c|}
\hline Dimensión & Ítems & Respuesta & Descriptivos \\
\hline $\begin{array}{l}\text { Estatus Subjeti- } \\
\text { vo }\end{array}$ & $\begin{array}{l}\text { En nuestra sociedad, hay grupos } \\
\text { que tienden a ubicarse en los nive- } \\
\text { les más altos y grupos que tienden } \\
\text { a ubicarse en los niveles más bajos } \\
\text { de la sociedad. ¿Dónde se ubicaría } \\
\text { Ud.? }\end{array}$ & $\begin{array}{l}1 \text { (más bajo) a } 10 \text { (más } \\
\text { alto) }\end{array}$ & $\begin{array}{l}\mathrm{M}=4.15 \\
\mathrm{SD}=1.59\end{array}$ \\
\hline $\begin{array}{l}\text { Ingreso familiar } \\
\text { per cápita }\end{array}$ & $\begin{array}{l}\text { Ingreso familiar (en pesos } \\
\text { chilenos) / número de integrantes } \\
\text { por hogar }\end{array}$ & & $\begin{array}{l}\mathrm{M}=218.028 \\
\mathrm{SD}=261.323\end{array}$ \\
\hline \multirow{5}{*}{ Educación } & \multirow{5}{*}{ ¿Cuál es su nivel educacional? } & $\begin{array}{l}\text { 1. Básica Completa o me- } \\
\text { nor }\end{array}$ & $11.79 \%$ \\
\hline & & $\begin{array}{l}\text { 2. Básica completa o Me- } \\
\text { dia incompleta }\end{array}$ & $21.41 \%$ \\
\hline & & 3. Media completa & $27.54 \%$ \\
\hline & & $\begin{array}{l}\text { 4. Superior no universita- } \\
\text { ria o universitaria incom- } \\
\text { pleta }\end{array}$ & $25.20 \%$ \\
\hline & & $\begin{array}{l}\text { 5.Universitaria completa } \\
\text { o superior }\end{array}$ & $14.04 \%$ \\
\hline
\end{tabular}

Finalmente, el tercer set de variables se relaciona con la percepción de desigualdad económica. En los modelos estas variables se estiman como dependientes tanto de las variables de meritocracia como también de las variables de estatus. Los dos indicadores a analizar son una medida de la percepción general de la magnitud de la desigualdad del ingreso en Chile, y otra más especifica sobre la desigualdad de salarios. Esta última se obtiene de manera indirecta utilizando dos preguntas del cuestionario: una referida a cuánto cree que gana un obrero (en pesos - moneda local) y la otra sobre cuál es el salario de un gerente. Con estas dos respuestas se realiza una proporción simple, dividiendo el ingreso del gerente por el ingreso del obrero. Por lo tanto, un valor de 10 expresa que el entrevistado 
considera que un gerente gana 10 veces lo que gana un obrero. A esta variable la llamamos "brecha percibida". Luego, a esta magnitud se le aplica un término logarítmico, siguiendo las recomendaciones de la literatura para el tratamiento de las variables de ingreso. El detalle se presenta en la Tabla 3.

Tabla 3: Variables de Desigualdad Percibida

\begin{tabular}{|c|c|c|c|}
\hline Dimensión & Ítems & Respuesta & Descriptivos \\
\hline $\begin{array}{l}\text { Percepción general de la desi- } \\
\text { gualdad }\end{array}$ & $\begin{array}{l}\text { ¿Qué piensa acerca de las } \\
\text { diferencias de ingreso en } \\
\text { Chile? Pienso que son... }\end{array}$ & $\begin{array}{l}\text { 1. Muy grandes } \\
\text { a } \\
\text { 5. Muy peque- } \\
\text { ñas * }\end{array}$ & $\mathrm{M}=1.37$ \\
\hline \multirow{2}{*}{ Brecha salarial percibida } & $\begin{array}{l}\text { ¿Cuánto dinero cree Ud. } \\
\text { que gana al mes el presi- } \\
\text { dente de una gran empre- } \\
\text { sa nacional? }\end{array}$ & \multirow{2}{*}{$\begin{array}{l}\text { Monto en pesos } \\
\text { chilenos }(\$)\end{array}$} & $\begin{array}{l}\mathrm{M}=14,460,975.61 \\
\mathrm{SD}=14,235,079.88 \\
\text { Mediana }=10,000,000.00\end{array}$ \\
\hline & $\begin{array}{l}\text { ¿Cuánto dinero cree Ud. } \\
\text { que gana al mes un obrero } \\
\text { no calificado de una fábri- } \\
\text { ca? }\end{array}$ & & $\begin{array}{l}\mathrm{SD}=115,796.58 \\
\text { Mediana }=210,000.00\end{array}$ \\
\hline
\end{tabular}

\footnotetext{
* Este ítem será invertido en los siguientes análisis para que valores mayores se asocien a mayor percepción de desigualdad (1=Muy pequeñas, $5=$ Muy grandes).
}

\section{Método}

Los análisis de los datos se realizan mediante la estimación de una serie de modelos con ecuaciones estructurales, debido principalmente al tratamiento del error de medición en modelos con variables latentes. Para ello se utilizó la librería lavaande $\mathrm{R}$, versión 0.5-20 (Rosseel, 2012).

\section{Resultados}

El primer paso del análisis se relaciona con la hipótesis de medición de meritocracia, a saber, que percepciones y preferencias constituyen aspectos distintos pero relacionados de este constructo. Para esto se utilizó la técnica de análisis factorial confirmatorio, la que permite señalar si el ajuste del modelo propuesto teóricamente es satisfactorio respecto de los datos. 
El modelo de medición de las variables latentes y el ajuste observado se muestran en la Figura 2. Cada uno de los ítems fue respondido en una escala ordinal de 5 niveles, razón por la cual el estimador general utilizado fue WLSMV (Weighted least square mean variance adjusted). En general, se observa que el modelo de medición planteado se encuentra dentro de los valores esperados para considerar un ajuste como adecuado ( $\mathrm{CFI}=0.99$; TLI=0.99; RMSEA $=0.052$ ).

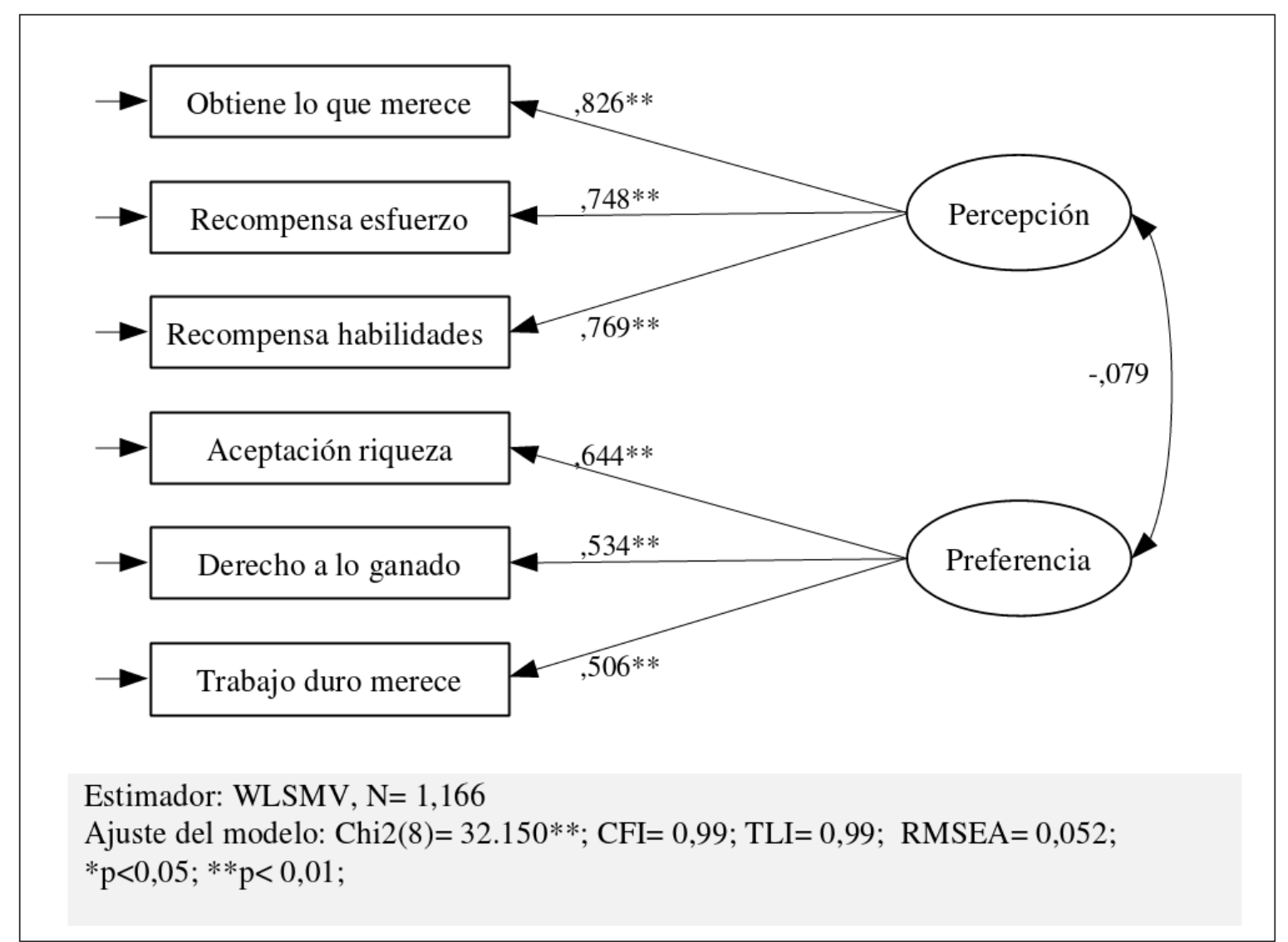

Figura 2: Analisis factorial confirmatorio percepciones y preferencias por meritocracia.

Respecto de las hipótesis planteadas para el modelo de medición, la meritocracia como constructo efectivamente presenta un comportamiento que permite distinguir sus aspectos descriptivos (percepción de meritocracia) de sus aspectos normativos (preferencia en la meritocracia), cargando cada uno de los set de tres indicadores en la dimensión latente respectiva. Resulta relevante destacar que se logró pesquisar una asociación entre ambas dimensiones de la meritocracia, sin embargo, esta es pequeña y no estadísticamente significativa $(\mathrm{r}=-.08, \mathrm{p}>.05)$. Por lo tanto, desde estos análisis existe evidencia para plantear 
que percepciones y preferencias son constructos distintos de la meritocracia y corresponde analizarlos por separado.

\section{Modelos de regresión}

La Tabla 4 muestra los resultados de la estimación de los modelos de regresión en contexto SEM, donde percepción y preferencia en la meritocracia son regresados como variables latentes en variables de estatus socioeconómico. Se presentan tres modelos para cada variable dependiente, el primero de ellos incluye educación e ingreso como medidas de estatus objetivo, el segundo agrega un índice que combina ingreso y educación relativa al grupo de referencia (a detallar posteriormente), y finalmente en el tercero se añade el indicador de estatus subjetivo. En todos los modelos se cumplen criterios satisfactorios de ajuste (Kline, 2011).

Un primer elemento a atender en el Modelo 1 de la Tabla 4 es la diferencia general entre los modelos para percepción y preferencias en meritocracia, dada la ausencia de efectos significativos para esta última variable. En términos más bien técnicos esto se puede relacionar con lo que ya se venía observando desde los datos descriptivos: los indicadores de preferencias en meritocracia poseen en general un mayor promedio y una menor varianza. Por lo tanto, las preferencias en la meritocracia aparecen como más consensuales, y dado que a menor varianza existe también menor covarianza, se presentan menos diferencias entre individuos y grupos reflejados en los efectos de las variables independientes. Ahora bien, en relación a lo que sucede con la percepción de meritocracia observamos que el ingreso posee un efecto positivo mientras el aumento en el nivel educacional se relaciona con una disminución en la percepción de meritocracia. El que estas variables posean efectos opuestos es sin duda interesante ya que por un lado nos indica que, ceteris paribus, el aumento neto de los ingresos conduce a percibir una mejor implementación del ideal meritocratico. No obstante, la percepción de meritocracia parece disminuir con el aumento del nivel educacional, lo que podría vincularse con los argumentos de Baer y Lambert (1982) de que los mayores niveles educacionales conllevarían una actitud más crítica respecto a la distribución de bienes en la sociedad. Una explicación alternativa a este mismo fenómeno estaría basada en la teoría de la deprivación relativa (Crosby, 1979; Walker y Pettigrew, 1984) ya que personas de un mayor estatus vía nivel educacional aspirarían a un mayor ingreso, situación que los podría hacer sentirse deprivados relativamente respecto a su grupo de referencia. Para poder explorar esta asociación se definió una variable que expresa la diferencia del ingreso de cada individuo respecto del promedio de ingresos del nivel educacional al cual pertenece, la que se agrega en el Modelo 2. Como vemos, esta variable 
captura la mayor parte de los efectos de ingreso y educación, sugiriendo que procesos de comparación social con el grupo de referencia de estatus podrían influir negativamente en la percepción de meritocracia. Si bien esta asociación no se encontraba prevista desde la fundamentación inicial, su inclusión de manera exploratoria permite delinear posibles ámbitos de investigación futura.

En relación al estatus subjetivo que se agrega en el Modelo 3, se observa que a una mayor autovaloración de la posición social, aumenta la percepción de meritocracia. En general estos resultados van en línea con la hipótesis del interés racional, ya que quienes consideran que están en una posición superior, tenderán a percibir que en la sociedad la implementación del ideal meritocrático funciona de mejor manera. Por lo tanto, más allá de los indicadores objetivos como educación e ingreso, lo que las personas piensan respecto de ellas mismas en términos de jerarquía social resulta relevante para su visión del funcionamiento de la meritocracia. 
Tabla 4: Percepción y preferencia en meritocracia en variables de estatus social

\begin{tabular}{|c|c|c|c|c|c|c|}
\hline & \multicolumn{2}{|c|}{ Modelo 1} & \multicolumn{2}{|c|}{ Modelo 2} & \multicolumn{2}{|c|}{ Modelo 3} \\
\hline & Percepción & Pref. & Percepción & Pref. & Percepción & Pref. \\
\hline $\begin{array}{l}\text { Ingreso familiar per } \\
\text { cápita }\end{array}$ & $\begin{array}{c}0.090^{* *} \\
(2.325)\end{array}$ & $\begin{array}{r}0.022 \\
(0.618)\end{array}$ & $\begin{array}{l}0.011 \\
(1.556)\end{array}$ & $\begin{array}{r}0.000 \\
(-0.031)\end{array}$ & $\begin{array}{c}-0.011 \\
(-1.133)\end{array}$ & $\begin{array}{r}0.000 \\
(-0.041)\end{array}$ \\
\hline \multicolumn{7}{|l|}{$\begin{array}{l}\text { Educación (ref=basica } \\
\text { inc.) }\end{array}$} \\
\hline $\begin{array}{l}\text { Básica completa o } \\
\text { media incompleta }\end{array}$ & $\begin{array}{r}-0.078 \\
(-0.728)\end{array}$ & $\begin{array}{r}0.073 \\
(0.654)\end{array}$ & $\begin{array}{r}-0.055 \\
(-0.500)\end{array}$ & $\begin{array}{c}0.081 \\
(0.715)\end{array}$ & $\begin{array}{c}-0.071 \\
(-0.650)\end{array}$ & $\begin{array}{c}0.093 \\
(0.802)\end{array}$ \\
\hline Media completa & $\begin{array}{l}-0.301^{* *} \\
(-2.898)\end{array}$ & $\begin{array}{r}0.018 \\
(0.172)\end{array}$ & $\begin{array}{l}-0.261^{* *} \\
(-2.469)\end{array}$ & $\begin{array}{r}0.030 \\
(0.281)\end{array}$ & $\begin{array}{l}-0.298^{* *} \\
(-2.797)\end{array}$ & $\begin{array}{r}0.051 \\
(0.458)\end{array}$ \\
\hline $\begin{array}{l}\text { Técnico completa y } \\
\text { sup. incompleta }\end{array}$ & $\begin{array}{l}-0.265^{*} \\
(-2.424)\end{array}$ & $\begin{array}{r}0.019 \\
(0.173)\end{array}$ & $\begin{array}{c}-0.192 \\
(-1.749)\end{array}$ & $\begin{array}{c}0.042 \\
(0.383)\end{array}$ & $\begin{array}{l}-0.247^{*} \\
(-2.217)\end{array}$ & $\begin{array}{r}0.077 \\
(0.678)\end{array}$ \\
\hline $\begin{array}{l}\text { Superior completa y } \\
\text { más }\end{array}$ & $\begin{array}{l}-0.280^{*} \\
(-2.166)\end{array}$ & $\begin{array}{c}-0.032 \\
(-0.247)\end{array}$ & $\begin{array}{r}-0.170 \\
(-1.347)\end{array}$ & $\begin{array}{r}0.000 \\
(0.003)\end{array}$ & $\begin{array}{l}-0.265^{*} \\
(-2.038)\end{array}$ & $\begin{array}{r}0.055 \\
(0.412)\end{array}$ \\
\hline $\begin{array}{l}\text { Ingreso - prom. ingresos } \\
\text { según educación }\end{array}$ & & & $\begin{array}{c}0.078^{*} \\
(1.986)\end{array}$ & $\begin{array}{r}0.021 \\
(0.568)\end{array}$ & $\begin{array}{r}0.072 \\
(1.823)\end{array}$ & $\begin{array}{c}0.031 \\
(0.856)\end{array}$ \\
\hline Estatus Subjetivo & & & & & $\begin{array}{l}0.074^{* *} \\
(3.765)\end{array}$ & $\begin{array}{r}-0.025 \\
(-1.236)\end{array}$ \\
\hline \multicolumn{7}{|l|}{ Ajuste } \\
\hline Chisq (df) & \multicolumn{2}{|c|}{$49.015(28)^{* *}$} & \multicolumn{2}{|c|}{$52.698(32)^{*}$} & \multicolumn{2}{|c|}{$58.581(36)^{*}$} \\
\hline CFI & \multicolumn{2}{|c|}{0.99} & \multicolumn{2}{|c|}{0.99} & \multicolumn{2}{|c|}{0.993} \\
\hline RMSEA & \multicolumn{2}{|c|}{0.028} & \multicolumn{2}{|c|}{0.026} & \multicolumn{2}{|c|}{0.026} \\
\hline
\end{tabular}

${ }^{*} \mathrm{p}<0.05 ;{ }^{* *} \mathrm{p}<0,01 ;$ valores $\mathrm{z}$ entre paréntesis. 


\section{Meritocracia y percepción de desigualdad}

En esta tercera parte del análisis nos abocaremos a los posibles efectos de las percepciones y preferencias en la meritocracia sobre la percepción de desigualdad, los que se presentan en la Tabla 5.

Tabla 5: Regresión de percepción de desigualdad económica en meritocracia y estatus

\begin{tabular}{|c|c|c|c|c|}
\hline & \multicolumn{2}{|c|}{ Modelo 1} & \multicolumn{2}{|c|}{ Modelo 2} \\
\hline & Salarial & General & Salarial & General \\
\hline Percepción meritocracia & $\begin{array}{l}-0.165^{* *} \\
(-3.492)\end{array}$ & $\begin{array}{c}-0.140^{* *} \\
(-4.217)\end{array}$ & $\begin{array}{c}-0.176^{* *} \\
(3.433)\end{array}$ & $\begin{array}{c}-0.116^{* *} \\
(-3.272)\end{array}$ \\
\hline Preferencia meritocracia & $\begin{array}{l}0.262^{* *} \\
(3.346)\end{array}$ & $\begin{array}{l}0.200^{* *} \\
(3.279)\end{array}$ & $\begin{array}{c}0.296^{* *} \\
(3.411)\end{array}$ & $\begin{array}{c}0.224^{* *} \\
(3.310)\end{array}$ \\
\hline Ingreso familiar per cápita & & & $\begin{array}{l}0.097 \\
(1.930)\end{array}$ & $\begin{array}{c}-0.011 \\
(-0.273)\end{array}$ \\
\hline $\begin{array}{l}\text { Educación (ref=basica y media } \\
\text { incompleta.) }\end{array}$ & & & $\begin{array}{c}0.092 \\
(0.696)\end{array}$ & $\begin{array}{c}0.042 \\
(0.452)\end{array}$ \\
\hline Media completa & & & $\begin{array}{c}0.173 \\
(1.347)\end{array}$ & $\begin{array}{l}0.213^{*} \\
(2.231)\end{array}$ \\
\hline $\begin{array}{l}\text { Técnico completa y sup. } \\
\text { incompleta }\end{array}$ & & & $\begin{array}{l}0.120 \\
(0.894)\end{array}$ & $\begin{array}{l}0.225^{*} \\
(2.309)\end{array}$ \\
\hline Superior completa y más & & & $\begin{array}{l}0.162 \\
(0.949)\end{array}$ & $\begin{array}{l}0.343^{*} \\
(2.281)\end{array}$ \\
\hline Estatus Subjetivo & & & $\begin{array}{c}-0.011 \\
(-0.430)\end{array}$ & $\begin{array}{l}-0.064^{* *} \\
(-3.863)\end{array}$ \\
\hline Ajuste & & & & \\
\hline $\begin{array}{l}\text { Chisq (df) } \\
C F I \\
R M S E A\end{array}$ & & $\begin{array}{c}146.738(52)^{* *} \\
0.967 \\
0.046\end{array}$ & & $\begin{array}{c}33.532(16)^{* *} \\
0.994 \\
0.034\end{array}$ \\
\hline
\end{tabular}

${ }^{*} \mathrm{p}<0.05 ;{ }^{*} \mathrm{p}<0,01 ;$ valores z entre paréntesis.

En el Modelo 1 de la Tabla 5 se ingresan las variables de percepciones y preferencias en la meritocracia ahora como independientes de las dos variables de percepción de desigualdad: percepción de desigualdad salarial (en base a la proporción de ingresos percibidos para gerente y obrero) y percepción de desigualdad general relacionada con la 
magnitud percibida de las diferencias de ingreso en el país, desde muy bajas hasta muy altas. Aquí se observan efectos consistentes de ambas variables de meritocracia en las dos medidas de desigualdad percibida: positiva para el caso de las preferencias, y negativa para el caso de la percepción. Es decir, a mayor preferencia por meritocracia se percibe más desigualdad económica y a mayor percepción de meritocracia se percibe menos desigualdad económica. En este caso la preferencia por meritocracia estaría expresando, además de una opción normativa, una demanda por redistribución vinculada a la mayor percepción de desigualdad. Por su parte, la percepción de que el ideal meritocrático se implementa adecuadamente conllevaría una cierta legitimación de las diferencias de ingreso, ya que se debería a razones más bien individuales que sistémicas. Un aspecto técnico que contextualiza y también releva este hallazgo es que la correlación entre las dos medidas de percepción de desigualdad es baja $(\mathrm{r}=0.054, \mathrm{p}=0.027)$, por lo tanto las asociaciones similares con las variables de meritocracia no son un mero artefacto metodológico sino que se asocian con indicadores que estarían capturando aspectos distintos de un mismo fenómeno.

El Modelo 2 de la Tabla 5 agrega las variables de estatus objetivo y subjetivo que analizamos en la Tabla 5. Observamos que los efectos de percepción y preferencias en la meritocracia se mantienen controlando por variables de estatus, y que éstas presentan efectos diferenciales en percepción general y percepción de desigualdad salarial. Al respecto, la variable ingreso es la que muestra un efecto positivo en percepción de desigualdad salarial, mientras el nivel educacional universitario y el estatus subjetivo se asocian positivamente con desigualdad general. Si bien estos efectos no son parte esencial de las hipótesis de este trabajo, es interesante notar esta asociación diferencial para ambas variables dependientes de percepción, que además van en línea con investigaciones anteriores que relacionan un mayor estatus a más desigualdad percibida (Castillo, 2012; Wegener, 1990).

\section{Discusión}

La preocupación creciente por las desigualdades ha dado realce a interrogantes sobre cómo es posible la convivencia democrática con extremas disparidades económicas, por qué existen distintos niveles de presión redistributiva en ese contexto, o por qué formas la desigualdad es justificada. Los resultados de este artículo ilustran al menos en parte la contribución que el análisis específico del mérito y la meritocracia puede hacer a estas discusiones, enfocándose en las distinciones entre lo que se prefiere y lo que se percibe, como también en la posición relativa del individo en la estructura social. Esto permite comprender más precisamente la interacción entre desigualdad y meritocracia en las sociedades actuales, como también por qué existen distintos niveles de tolerancia a la desigualdad y de valoración del logro frente a la adscripción". 
Si bien la meritocracia es un concepto mencionado frecuentemente en investigaciones y estudios sociológicos principalmente en relación con desigualdad y estratificación social, en general aparece como un concepto auxiliar y son pocas las investigaciones que se han enfocado en la discusión y estudio empírico de este concepto en sí mismo. En este artículo se propuso un marco general para los estudios de meritocracia, distinguiendo perspectivas normativas, descriptivas y subjetivas, para luego enfocarse en la perspectiva subjetiva, su medición y sus posibles implicancias.

Un primer aspecto a discutir del trabajo empírico de medición, relacionado con la primera hipótesis general, es que es posible identificar dos dimensiones distintas en la perspectiva subjetiva de la meritocracia, a saber, percepciones y preferencias. Estas dos dimensiones poseen una correlación muy baja, y por lo tanto se justifica su consideración como dos objetos distintos de estudio. Así, el que se perciba un buen funcionamiento de la meritocracia no necesariamente se relaciona con que se considere que este sistema es el mejor, y por lo tanto sería un error concluir un apoyo normativo a la meritocracia desde evaluaciones de su funcionamiento. Nos parece que este avance en temas de medición asociada a teoría mediante técnicas confirmatorias permite dar luces sobre conceptos que hasta ahora habían sido evaluados de manera exploratoria, con supuestos no contrastados respecto a la estructura latente del modelo (Duru-Bellat \& Tenret, 2012), y muchas veces confundiendo percepciones con preferencias bajo el término general de creencias. Si bien el énfasis en la medición de conceptos es algo que aparece más frecuentemente en disciplinas como la psicología y educación, constituye un área de desarrollo conceptual y metodológico que se abre campo en la sociología dadas sus posibilidades de contraste de teorías y evaluación de comparabilidad en distintas poblaciones (Davidov et al., 2014). Por lo tanto, esta investigación sirve como referencia para futuras aproximaciones empíricas en el estudio de la meritocracia.

Un aspecto que marca diferencias entre las mediciones de percepciones y preferencias en meritocracia son sus niveles (promedio) y su variabilidad. Mientras que en general se percibe un bajo funcionamiento en la meritocracia pero con alta variabilidad, las preferencias se mantienen en un nivel mayor y también con más consenso. Esto, sumado a la falta de asociación de percepciones y preferencias, podría estar indicando que la preferencia por la meritocracia parece ser bastante resiliente a las potenciales carencias percibidas en su funcionamiento. De otra manera, aunque se perciba poca meritocracia en la sociedad, se puede todavía mantener la creencia de que es un sistema apropiado para la distribución de recursos. Este aspecto afirma las concepciones sobre la función legitimadora de la meritocracia, pero sin embargo también se abre la pregunta sobre si existe algún punto crítico en el cual el funcionamiento de la meritocracia podría comenzar a afectar las 
preferencias, lo cual requeriría ampliar la investigación a técnicas experimentales y también a comparación internacional.

La mayor parte de las hipótesis que relacionan variables de estatus social con meritocracia pudieron ser analizadas solo en relación a percepción de meritocracia, ya que el carácter más consensual de las preferencias (vis-a-vis su menor varianza) impidió detectar diferencias significativas. En principio se observaron efectos opuestos para las variables de ingreso y educación en percepción de meritocracia, positivos y negativos respectivamente. Esto sugiere una hipótesis interesante a contrastar en futuros estudios, a saber, que el mayor ingreso puede ser asociado a esfuerzo individual y que por tanto la percepción de que la meritocracia posee un buen funcionamiento en la sociedad podría vincularse a la justificación de la desigualdad. Este hallazgo se refuerza con la variable de estatus subjetivo, ya que quienes se autoperciben ocupando posiciones superiores en la escala social también perciben un mejor funcionamiento de la meritocracia. Por el contrario, la relación negativa de la educación con percepción de meritocracia (controlando por ingreso) podría estar indicando una situación de deprivación relativa, donde personas que se han esforzado por alcanzar mayores credenciales no verían recompensados sus esfuerzos y/o talentos. Futuras investigaciones que consideren medidas adicionales de comparación de ingresos podrían arrojar más luces sobre este punto.

Respecto a las hipótesis que asocian percepciones y preferencias en meritocracia con percepción de desigualdad económica, los hallazgos sugieren un ámbito fructífero para futuros estudios. Consistentemente, tomando en cuenta dos medidas distintas de percepción de desigualdad, a mayor percepción de meritocracia menos se percibe la desigualdad económica, y mientras más se cree en la meritocracia, más desigualdad se percibe. Por lo tanto, percepciones y preferencias poseen asociaciones opuestas en relación a un mismo fenómeno, sugiriendo una mayor tolerancia y legitimación de las diferencias económicas para aquellos que perciben un adecuado funcionamiento de la distribución basada en mérito, mientras que la mayor preferencia llevaría a un estándar de más exigencia frente a la realidad y consistentemente a una demanda por una reducción de las diferencias que podrían basarse en criterios distintos al mérito.

Existen una serie de limitaciones en esta investigación que podrían ser consideradas en futuros estudios. Una de ellas tiene que ver con los instrumentos utilizados para la medición de meritocracia, que si bien son parte de una encuesta especializada en temas de justicia distributiva, no permiten operacionalizar de manera muy específica los constructos estudiados, principalmente el de preferencias. Idealmente, los indicadores de preferencias deberían ser "espejo" de los de percepción, en el primer caso preguntando por lo que debería 
ser (preferencias) y en el segundo por lo que sucede realmente en la sociedad estudiada (percepción), pero respecto a un mismo tema. Si bien el modelo propuesto en esta investigación funciona bien en términos métricos, se requiere seguir avanzando en la precisión de los indicadores. Una segunda limitación es que el estudio se refiere solo a un país, caracterizado por una alta desigualdad económica y políticas neoliberales en ámbitos tradicionales de estado de bienestar como son educación, salud y educación. Intencionalmente, decidimos no poner esto como un contexto inicial ya que este estudio no pretende ser un caso aplicado específicamente a un país, sino generar discusión conceptual y de medición más general. Además, en ausencia de comparación internacional no es posible afirmar que los resultados son generalizables a otros contextos. Por lo tanto, las justificaciones del o los casos a analizar adquieren mucho más sentido en estudios comparativos por desarrollar en el futuro.

Desde esta investigación se abren una serie de posibles ámbitos de profundización en el estudio empírico de la meritocracia, de los cuales se podrían señalar algunos. En primer lugar, seguir abordando en el ámbito de los instrumentos de medición, mediante el diseño y prueba de otros indicadores así como también estimar la equivalencia de las mediciones entre distintas poblaciones/países mediante procedimientos de invarianza métrica (Davidov et al., 2014) Segundo, los elementos constitutivos más tradicionales de la meritocracia, como son esfuerzo y talento, no han sido estudiados por separado a la fecha. El contar con medidas más específicas para ambos, y en combinación con las dimensiones perceptual y de preferencias, abre una serie de posibilidades para explorar nuevas características de los aspectos subjetivos de la meritocracia. En tercer lugar, la relación entre variables de estatus socioeconómico y meritocracia está lejos de delinear un escenario claro y lineal. Tenemos evidencia que ellas se relacionan principalmente con percepciones de meritocracia, pero en general de manera inversa, siendo lo más interesante el indicio de una relación negativa entre mayor educación y menor percepción de meritocracia. Esto puede deberse a fenómenos de deprivación relativa, al efecto ilustrador de la educación, y/o a algún otro motivo que merece mayor profundización. Cuarto, es necesario reconocer la complejidad del fenómeno de la meritocracia y acompañar estas investigaciones basadas en encuestas con estudios de tipo cualitativo que permitan dar nuevas luces sobre los significados de este concepto y discutir con la evidencia cuantitativa. Finalmente, además de relacionar meritocracia con variables de percepción de desigualdad es posible pensar en otros fenómenos relevantes asociados, como son las preferencias distributivas: ¿Son aquellos que perciben un buen funcionamiento de la meritocracia más aversos a redistribuir por parte del estado? El vínculo con la literatura de preferencias, que posee un amplio desarrollo en economía y ciencia política, permitirá cubrir 
una brecha conceptual y disciplinar interesante para poder comprender de mejor manera fenómenos distributivos en sociedades modernas. 


\section{Referencias}

Aalberg T. 2003. Achieving Justice: Comparative Public Opinion on Income Distribution. Leiden: Brill.

Adams J. 1963. Towards an Understanding of Inequity. Journal of Abnormal Psychology 67(5): 422-36. https://doi.org/10.1037/h0040968

Alon S. y Tienda M. 2007. Diversity, Opportunity, and the Shifting Meritocracy in Higher Education. American Sociological Review 72(4): 487-511. https://doi.org/10.1177/000312240707200401

Aristotle 1999. Nichomaquean Ethics. Kitchener: Batoche Books.

Baer D. y Lambert R. 1982. Education and support for dominant ideology. Canadian Review of Sociology/Revue canadienne de sociologie 19(2): 173-195. https://doi.org/10.1111/j.1755-618X.1982.tb00859.x

Bell D. 1977. Las contradicciones culturales del capitalismo. México: Alianza.

Bourdieu P. y Passeron J. 1990- Reproduction in education, society, and culture. 1990 ed. Theory, culture \& society, London; Newbury Park, Calif: Sage in association with Theory, Culture \& Society, Dept. of Administrative and Social Studies, Teesside Polytechnic.

Breen R. y Goldthorpe J. H. 2001. Class, mobility and merit the experience of two British birth cohorts. European sociological review 17(2): 81-101. https://doi.org/10.1093/esr/17.2.81

Castillo J.C. 2012. Is inequality becoming just? Changes in public opinion about economic inequality in Chile. Bulletin of Latin American Research 31(1): 1-18. https://doi.org/10.1111/j.1470-9856.2011.00605.x

Encuesta Proyecto FONDECYT "Justicia social y participación ciudadana" (2014). Datos disponibles por solicitud a jcastillov@uc.cl. 
Costa-Lopes R., Dovidio J. F., Pereira C.R., et al. 2013. Social psychological perspectives on the legitimation of social inequality: Past, present and future. European Journal of Social Psychology 43(4): 229-237. https://doi.org/10.1002/eisp.1966

Crosby F. 1979. Relative deprivation revisited: A response to Miller, Bolce, and Halligan. The American Political Science Review 73(1): 103-112. https://doi.org/10.2307/1954733

Davidov E., Meuleman B., Cieciuch J-, et al. 2014. Measurement Equivalence in CrossNational Research. Annual Review of Sociology 40(1): 55-75. https://doi.org/10.1146/annurev-soc-071913-043137

Deutsch M. 1975. Equity, Equality, and Need: What Determines Which Value Will Be Used as a Basis of Distributive Justice? Journal of Social Issues 31(3): 137-150. https://doi.org/10.1111/j.1540-4560.1975.tb01000.x

Duru-Bellat M. y Tenret E. 2012. Who's for Meritocracy? Individual and Contextual Variations in the Faith. Comparative Education Review 56(2): 223-247. https://doi.org/10.1086/661290

Foster M. D. y Tsarfati E. M. 2005. The Effects of Meritocracy Beliefs on Women's WellBeing After First-Time Gender Discrimination. Personality and Social Psychology Bulletin 31(12): 1730-1738. https://doi.org/10.1177/0146167205278709

Goldthorpe, J. H. 2003. The myth of education-based meritocracy. New Economy 10(4): 234239. https://doi.org/10.1046/i.1468-0041.2003.00324.x

Hadjar, A. 2008. Meritokratie als Legitimationsprinzip. Wiesbaden: VS Verlag.

Homans, G. 1974. Social behavior: Its elementary forms. New York: Harcouit Brace Jovanovich.

Horowitz, I. L. 2006. The Moral Economy of Meritocracy: or, the Unanticipated Triumph of Reform and the Failure of Revolution in the West. The Political Quarterly 77: 127133. https://doi.org/10.1111/i.1467-923X.2006.00789.x

Jaime-Castillo, A.M. y Marqués-Perales, I. 2014. Beliefs about Social Fluidity and Preferences for Social Policies. Journal of Social Policy 43(3): 615-633. https://doi.org/10.1017/S0047279414000221

Janmaat, J.G. 2013. Subjective inequality: A review of international comparative studies on people's views about inequality. European Journal of Sociology 54(3): 357-389. https://doi.org/10.1017/S0003975613000209 
Jost, J., Blount, S., Pfeffer, J., et al. 2003. Fair Market Ideology: Its Cognitive-Motivational Underpinnings. Research in Organizational Behavior 25: 53-91. https://doi.org/10.1016/S0191-3085(03)25002-4

Kline, R. 2011. Principles and practice of structural equation modeling. 2nd ed. New York: Guilford Press.

Kluegel, J. y Smith, E. 1981. Beliefs About Stratification. Annual Review of Sociology 7: 2956. https://doi.org/10.1146/annurev.so.07.080181.000333

Krebs, A. 2000. Gleichheit oder Gerechtigkeit. Frankfurt/Main: Suhrkamp.

Kunovich, S. y Slomczynski, K.M. 2007. Systems of Distribution and a Sense of Equity: A Multilevel Analysis of Meritocratic Attitudes in Post-industrial Societies. European Sociological Review 23(5): 649-663. https://doi.org/10.1093/esr/icm026

Lane, R. 1986. Market Justice, Political Justice. The American Political Science Review 80(2): 383-402. https://doi.org/10.2307/1958264

Lerner, M. 1980. The Belief in a Just World. A Fundamental Delusion. New York: Plenum Press. https://doi.org/10.1007/978-1-4899-0448-5

Lipset, S. 1959. Some Social Requisites of Democracy: Economic Development and Political Legitimacy. The American Political Science Review 53(1): 69-105. https://doi.org/10.2307/1951731

Meltzer, A. y Richard, S. 1981. A Rational Theory of the Size of Government. The Journal of Political Economy 89(5): 914. https://doi.org/10.1086/261013

Oxfam (2016) An economy for the one percent. Disponible en: https://www.oxfam.org/en/research/economy-1

Page, O. 2013. Mérito e igualdad de oportunidades. Revista de ciencia política (Santiago) 33(2): 533-545. https://doi.org/10.4067/S0718-090X2013000200006

Rawls, J. 1971. A Theory of Justice. Cambridge: Harvard University Press.

Piketty, T. (2014). Capital in the twenty-first century (A. Goldhammer, Trans.). Cambridge, MA: The Belknap Press of Harvard University Press. 
Reynolds, J. y Xian, H. 2014. Perceptions of meritocracy in the land of opportunity. Research in Social Stratification and Mobility 36: 121-137. https://doi.org/10.1016/i.rssm.2014.03.001

Rosseel, Y. (2012). lavaan: An R Package for Structural Equation Modeling. Journal of Statistical Software, 48(2), 1-36.

Sandel, M. 1998. Liberalism and the Limits of Justice. Cambridge: Cambridge University Press. https://doi.org/10.1017/CBO9780511810152

Schneider, S.M. and Castillo J.C. 2015. Poverty Attributions and the Perceived Justice of Income Inequality A Comparison of East and West Germany. Social Psychology Quarterly 78(3): 263-282. https://doi.org/10.1177/0190272515589298

Schröder, M. 2017. Is Income Inequality Related to Tolerance for Inequality? Social Justice Research 30(1): 23-47. https://doi.org/10.1007/s11211-016-0276-8

Sen, A. 2000. Merit and Justice. In: Arrow KJ, Bowles S, and Durlauf SN (eds), Meritocracy and economic inequality, Princeton, N.J: Princeton University Press, pp. 5-16.

Solt, F. 2010. Does Economic Inequality Depress Electoral Participation? Testing the Schattschneider Hypothesis. Political Behavior 32(2): 285-301. https://doi.org/10.1007/s11109-010-9106-0

Solt, F., Hu, Y., Hudson, K., et al. .2016. Economic inequality and belief in meritocracy in the United States. Research \& Politics 3(4): 2053168016672101. https://doi.org/10.1177/2053168016672101

Son Hing, L.S., Bobocel, D.R., Zanna, M.P., et al. 2011. The merit of meritocracy. Journal of Personality and Social Psychology 101(3): 433-450. https://doi.org/10.1037/a0024618

Stouffer, S., Suchman, E., Devinney, L., et al. 1949. The American Soldier. Princeton: Princeton University Press.

Tocqueville, A. 2004. El Antiguo Régimen y la Revolución. Ediciones AKAL.

Walker, I. y Pettigrew, T.F. 1984. Relative deprivation theory: an overview and conceptual critique. British Journal of Social Psychology 23: 301-310. https://doi.org/10.1111/i.20448309.1984.tb00645.x

Walzer, M. 1983. Spheres of Justice: A Defense of Pluralism and Equality. New York Oxford: Basic Books. 
Warikoo, N.K. y Fuhr, C. 2014. Legitimating status: perceptions of meritocracy and inequality among undergraduates at an elite British university. British Educational Research Journal 40(4): 699-717. https://doi.org/10.1002/berj.3108

Wegener, B. 1990. Equity, Relative Deprivation, and the Value Consensus Paradox. Social Justice Research 4(1): 65-86. https://doi.org/10.1007/BF01048536

Yair, G. 2007. Meritocracy. In: Ritzer G, The Blackwell Encyclopedia of Sociology, Oxford, UK, Malden, USA and Carlton, Australia: Blackwell Publishing Ltd. Available from: http://doi.wiley.com/10.1111/b.9781405124331.2007.x (accessed 15 July 2015).

Young, M. 1962. The Rise of the Meritocracy. Baltimore: Penguin Books. 